\section{TATRA \\ MOUNTaiNS \\ Mathematical Publications}

DOI: $10.2478 / \mathrm{tmmp}-2013-0016$

Tatra Mt. Math. Publ. 55 (2013), 27-36

\title{
ALGEBRAIC AND SET-THEORETICAL PROPERTIES OF SOME SUBSETS OF FAMILIES OF CONVERGENT AND DIVERGENT PERMUTATIONS
}

\author{
ROMAN WiTUŁA
}

\begin{abstract}
The paper presents a few basic algebraic and set-theoretical properties of some subsets of families of convergent and divergent permutations of $\mathbb{N}$, especially the compositions of families of the so-called one-sided convergent and one-sided divergent permutations.
\end{abstract}

\section{Introduction}

This paper is a sequel to [3]. We will discuss some inclusion relations for and between the families $\mathfrak{C} \mathfrak{D} \circ \mathfrak{D C}$ and $\mathfrak{D C} \circ \mathfrak{C} \mathfrak{D}$ as subsets of the family $\mathfrak{P}$ of all permutations of $\mathbb{N}$. Moreover, it will be determined for which permutations $p$ of $\mathbb{N}$ the following inclusions $p \mathfrak{D} p^{-1} \subseteq \mathfrak{D}$ and $p \mathfrak{D} \mathfrak{D} p^{-1} \subseteq \mathfrak{D} \mathfrak{D}$ hold true.

Permutation $p$ of $\mathbb{N}$ is convergent if for every convergent series $\sum a_{n}$ the $p$-rearranged series $\sum a_{p(n)}$ is also convergent. The family of all convergent permutations will be denoted by $\mathfrak{C}$. Permutations belonging to the family $\mathfrak{D}:=\mathfrak{P} \backslash \mathfrak{C}$ will be called the divergent permutations which is compatible with the following definition of divergent permutations.

Definition 1.1. A permutation $p$ of $\mathbb{N}$ is called a divergent permutation if there exists a conditionally convergent real series $\sum a_{n}$ such that the $p$-rearranged series $\sum a_{p(n)}$ is divergent.

In this paper only the series of real terms are discussed.

(C) 2013 Mathematical Institute, Slovak Academy of Sciences. 2010 Mathematics Subject Classification: 40A05, 05A99.

Keywords: convergent permutations, divergent permutations, one-sided convergent permutations, one-sided divergent permutations. 
Let $A, B \subset \mathfrak{P}$. Then the following family of permutations of $\mathbb{N}$

$$
\left\{p \in \mathfrak{P}: p \in A \text { and } p^{-1} \in B\right\}
$$

will be denoted by $A B$. After $\mathrm{Kron} \operatorname{rod}$ [1] and [2], we call:

a) elements of $\mathfrak{C} \mathfrak{C}$ - two-sided convergent permutations,

b) elements of $\mathfrak{C D}$ - one-sided convergent permutations,

c) elements of $\mathfrak{D C}$ - one-sided divergent permutations,

d) elements of $\mathfrak{D D}$ - two-sided divergent permutations.

Symbol o denotes here the composition of nonempty subsets of $\mathfrak{P}$, i.e.,

$$
B \circ A=\{q \circ p(\cdot):=q(p(\cdot)): q \in B \text { and } p \in A\}
$$

for any nonempty subsets $A, B$ of $\mathfrak{P}$. Operation $\circ$ is not commutative in general (which is rather obvious), but we have [3]

$$
\begin{gathered}
\mathfrak{D D} \circ \mathfrak{D C}=\mathfrak{D} \mathfrak{D} \cup \mathfrak{D C}=\mathfrak{D C} \circ \mathfrak{D} \mathfrak{D}, \\
\mathfrak{C} \circ \mathfrak{D D}=\mathfrak{C D} \circ \mathfrak{D D}=\mathfrak{C D} \cup \mathfrak{D D}=\mathfrak{D} \mathfrak{D} \circ \mathfrak{C D}=\mathfrak{D} \mathfrak{D} \circ \mathfrak{C} .
\end{gathered}
$$

However, it is interesting that $\mathfrak{D C} \circ \mathfrak{C} \mathfrak{D} \neq \mathfrak{C} \mathfrak{D} \circ \mathfrak{D C}$ as well. More precisely, in a separate paper [5] it was proved that the family $(\mathfrak{D C} \circ \mathfrak{C} \mathfrak{D}) \backslash(\mathfrak{C D} \circ \mathfrak{D C})$ is nonempty. We note that the family $\mathfrak{C} \mathfrak{C}$ is a unit element with respect to the composition with $\mathfrak{C}, \mathfrak{D}, \mathfrak{C} \mathfrak{C}, \mathfrak{C} \mathfrak{D}, \mathfrak{D C}, \mathfrak{D} \mathfrak{D}$, the finite compositions of these families and even the group $\mathfrak{G}$ generated by $\mathfrak{C}$.

We know (see [2], [3], 6], 7]) that permutation $p \in \mathfrak{P}$ is a convergent permutation if and only if there exists $c=c(p) \in \mathbb{N}$ such that for each interval $I$ of $\mathbb{N}$ the set $p(I)$ is a union of $c$ MSI. We say that a nonempty set $A \subset \mathbb{N}$ is a union of $n$ MSI (or of at most $n$ MSI) if there exists a family $\mathfrak{I}$ of $n$ (or at most $n$ ) intervals of $\mathbb{N}$ which form a partition of $A$ and $\operatorname{dist}(I, J) \geqslant 2$ for any two different members $I, J$ of $\mathfrak{J}$. MSI is the abbreviated form of the notion of mutually separated intervals.

Moreover, to denote the cardinality of set $G \subset \mathbb{N}$ we will use symbols $|G|$ or card $(G)$, with respect to the context of discussion.

\section{Main results}

The main results of this paper are listed below.

(i) The family $\mathfrak{P} \backslash \mathfrak{D} \mathfrak{D}$ is a proper subset of the family $(\mathfrak{C} \mathfrak{D} \circ \mathfrak{D C}) \cap(\mathfrak{D C} \circ \mathfrak{C} \mathfrak{D})$.

(ii) The family $(\mathfrak{C D} \circ \mathfrak{D C}) \cup(\mathfrak{D C} \circ \mathfrak{C D})$ is not equal to $\mathfrak{P}$.

(iii) The inclusion $p \mathfrak{D} p^{-1} \subseteq \mathfrak{D}\left(p \mathfrak{D} \mathfrak{D} p^{-1} \subseteq \mathfrak{D} \mathfrak{D}\right.$, resp.) holds true if and only if $p \in \mathfrak{C} \mathfrak{C}$. 


\section{CONVERGENT AND DIVERGENT PERMUTATIONS}

(iv) The following relations

$$
(p \mathfrak{C} \mathfrak{C} q) \cap \mathfrak{D} \mathfrak{D} \neq \varnothing \quad \text { and } \quad(q \mathfrak{C} \mathfrak{C} p) \cap \mathfrak{D} \mathfrak{D} \neq \varnothing,
$$

for any $p \in(\mathfrak{C D} \cup \mathfrak{D D})$ and $q \in \mathfrak{D}$ are fulfilled.

Let us start with the first announced result.

ThEOREM 2.1. The family $\mathfrak{P} \backslash \mathfrak{D} \mathfrak{D}$ is a proper subset of the family $(\mathfrak{C D} \circ \mathfrak{D C}) \cap$ $(\mathfrak{D C} \circ \mathfrak{C} \mathfrak{D})$.

P r o of. Let us denote by $\mathfrak{B}$ the family $(\mathfrak{C} \mathfrak{D} \circ \mathfrak{D C}) \cap(\mathfrak{D C} \circ \mathfrak{C} \mathfrak{D})$ and let $p \in \mathfrak{C} \mathfrak{C}$ and $q \in \mathfrak{C D}$. Then, we get $p q, q p \in \mathfrak{C D}$ (cf. 3. Theorem 2.6]) and therefore, $p=(p q) q^{-1}=q^{-1}(q p) \in \mathfrak{B}$, i.e., $\mathfrak{C} \mathfrak{C} \subset \mathfrak{B}$. Now, let $p \in \mathfrak{D C}$. Since $\mathfrak{D C} \circ \mathfrak{D C}=\mathfrak{D C}$, we obtain $p=(p p) p^{-1}=p^{-1}(p p) \in \mathfrak{B}$ and hence, $\mathfrak{D} \mathfrak{C} \subset \mathfrak{B}$. This implies that $\mathfrak{C D} \subset \mathfrak{B}$, too. In other words, the set $\mathfrak{P} \backslash \mathfrak{D D}$ is a subset of the family $\mathfrak{B}$.

Now, we give an example of commuting permutations $p \in \mathfrak{D C}$ and $q \in \mathfrak{C} \mathfrak{D}$ such that $p q \in \mathfrak{D D}$, which implies that $\mathfrak{B} \cap \mathfrak{D} \mathfrak{D} \neq \emptyset$, i.e., that $\mathfrak{P} \backslash \mathfrak{D} \mathfrak{D}$ is a proper subset of $\mathfrak{B}$.

Suppose the intervals $I_{n}, n \in \mathbb{N}$, form a partition of $\mathbb{N}$ and card $I_{n}=2 n$ for every $n \in \mathbb{N}$. Let us put

$$
\gamma\left(i+\min I_{n}\right)= \begin{cases}2 i+\min I_{n} & \text { for } \quad i=0,1, \ldots, n-1, \\ 2(i-n)+1+\min I_{n} & \text { for } \quad i=n, n+1, \ldots, 2 n-1,\end{cases}
$$

for each $n \in \mathbb{N}$. Next, we define the permutations $p$ and $q$ by setting

and

$$
p(i)=\gamma(i) \quad \text { and } \quad q(i)=i \quad \text { whenever } \quad i \in \bigcup_{n \in \mathbb{N}} I_{2 n}
$$

$$
p(i)=i \quad \text { and } \quad q(i)=\gamma^{-1}(i) \quad \text { whenever } \quad i \in \bigcup_{n \in \mathbb{N}} I_{2 n-1} .
$$

A careful reader could easily show that $p$ and $q$ have the desired properties.

Theorem 2.2. Let $p \in \mathfrak{P}$. Then we have

$$
p \notin \mathfrak{D C} \circ \mathfrak{C} \mathfrak{D} \Leftrightarrow(\mathfrak{C} \circ p) \subset \mathfrak{D} \mathfrak{D} \Leftrightarrow(p \circ \mathfrak{D C}) \subset \mathfrak{D} \mathfrak{D}
$$

and

$$
p \notin \mathfrak{C D} \circ \mathfrak{D C} \Leftrightarrow(p \circ \mathfrak{C}) \subset \mathfrak{D D} \Leftrightarrow(\mathfrak{D C} \circ p) \subset \mathfrak{D} \mathfrak{D} .
$$

P r o of. If $p \in \mathfrak{D C} \circ \mathfrak{C D}$, then $(\mathfrak{C} \mathfrak{D} \circ p) \cap \mathfrak{C} \mathfrak{D} \neq \varnothing$, i.e., $(\mathfrak{C} \circ p) \cap \mathfrak{C} \neq \varnothing$. Hence, if $(\mathfrak{C} \circ p) \subset \mathfrak{D D}$, then $p \notin \mathfrak{D C} \circ \mathfrak{C D}$. Now, if $p \in \mathfrak{D} \mathfrak{D}$ and $q p \in(\mathfrak{P} \backslash \mathfrak{D D})$ for some $q \in \mathfrak{C}$, then $q \in \mathfrak{C D}$ and $q p \in \mathfrak{C D}$ (cf. 3, Theorems 2.6 and 2.4]). Therefore, $p=q^{-1}(q p) \in \mathfrak{D C} \circ \mathfrak{C} \mathfrak{D}$, i.e., $p \in \mathfrak{D C} \circ \mathfrak{C} \mathfrak{D}$. On the other hand, by Theorem 2.1. the relation $p \notin \mathfrak{D C} \circ \mathfrak{C} \mathfrak{D}$ forces $p \in \mathfrak{D} \mathfrak{D}$. Summarizing the above arguments, we conclude that if $p \notin \mathfrak{D C} \circ \mathfrak{C D}$ then the inclusion $(\mathfrak{C} \circ p) \subset \mathfrak{D} \mathfrak{D}$ holds. So, we have proved that $(\mathfrak{C} \circ p) \subset \mathfrak{D D}$ if and only if $p \notin \mathfrak{D C} \circ \mathfrak{C D}$. 


\section{ROMAN WITUŁA}

The remaining relations

and

$$
\begin{aligned}
& p \notin \mathfrak{C D} \circ \mathfrak{D C} \quad \text { if and only if } \quad(p \circ \mathfrak{C}) \subset \mathfrak{D} \mathfrak{D}, \\
& p \notin \mathfrak{D C} \circ \mathfrak{C} \mathfrak{D} \quad \text { if and only if } \quad(p \circ \mathfrak{D C}) \subset \mathfrak{D} \mathfrak{D}
\end{aligned}
$$

$$
p \notin \mathfrak{C D} \circ \mathfrak{D C} \quad \text { if and only if }(\mathfrak{D C} \circ p) \subset \mathfrak{D} \mathfrak{D}
$$

may be shown in an analogous way.

Corollary 2.3. Let $p \in \mathfrak{P}$. Then

and

$$
p \notin \mathfrak{D C} \circ \mathfrak{C D} \Leftrightarrow\left(\mathfrak{C} \circ p^{-1}\right) \subset \mathfrak{D} \mathfrak{D} \Leftrightarrow\left(p^{-1} \circ \mathfrak{D C}\right) \subset \mathfrak{D} \mathfrak{D}
$$

$$
p \notin \mathfrak{C} \mathfrak{D} \circ \mathfrak{D C} \Leftrightarrow\left(p^{-1} \circ \mathfrak{C}\right) \subset \mathfrak{D} \mathfrak{D} \Leftrightarrow\left(\mathfrak{D C} \circ p^{-1}\right) \subset \mathfrak{D D} .
$$

Proof. It follows easily from two equalities

$$
(\mathfrak{D C} \circ \mathfrak{C D})^{-1}=\mathfrak{D C} \circ \mathfrak{C D} \quad \text { and }(\mathfrak{C D} \circ \mathfrak{D C})^{-1}=\mathfrak{C} \mathfrak{D} \circ \mathfrak{D C} \text {, }
$$

and then by using Theorem 2.2. Another proof can be obtained from the following four relations (cf. [3, Theorem 2.6]):

$$
\begin{aligned}
\left(p^{-1} \circ \mathfrak{D C}\right) & \subset \mathfrak{D D} \Leftrightarrow(\mathfrak{C} \circ p) \subset \mathfrak{D} \mathfrak{D}, \\
\left(\mathfrak{C} \circ p^{-1}\right) & \subset \mathfrak{D} \mathfrak{D} \Leftrightarrow(p \circ \mathfrak{D C}) \subset \mathfrak{D} \mathfrak{D}, \\
\left(p^{-1} \circ \mathfrak{C}\right) & \subset \mathfrak{D} \mathfrak{D} \Leftrightarrow(\mathfrak{D C} \circ p) \subset \mathfrak{D} \mathfrak{D}
\end{aligned}
$$

and

$$
\left(\mathfrak{D C} \circ p^{-1}\right) \subset \mathfrak{D} \mathfrak{D} \Leftrightarrow(p \circ \mathfrak{C}) \subset \mathfrak{D} \mathfrak{D} .
$$

Now, we present the example of a permutation $p \in \mathfrak{P}$ which is not an element of family $(\mathfrak{C D} \circ \mathfrak{D C}) \cup(\mathfrak{D C} \circ \mathfrak{C} \mathfrak{D})$. To prove this, we will apply Theorem 2.2,

ExAmple 1. Suppose the intervals $J_{k}^{(n)}, k, n \in \mathbb{N}, k \leqslant n$, with card $J_{k}^{(n)}=n$ and such that $J_{1}^{(n)}<J_{2}^{(n)}<\cdots<J_{n}^{(n)}<J_{1}^{(n+1)}$ form a partition of $\mathbb{N}$.

Define

$$
p\left(i-1+\min J_{k}^{(n)}\right)=k-1+\min J_{i}^{(n)}
$$

for any $i, k=1, \ldots, n$ and $n \in \mathbb{N}$. We are going to prove the following inclusion $(\mathfrak{C} \circ p) \cup(p \circ \mathfrak{C}) \subset \mathfrak{D} \mathfrak{D}$ which, in view of Theorem 2.2, is equivalent to the relation $p \notin((\mathfrak{C D} \circ \mathfrak{D C}) \cup(\mathfrak{D C} \circ \mathfrak{C D}))$.

First, we show that $p \circ \mathfrak{C} \subset \mathfrak{D D}$. Let $q \in \mathfrak{C}$. Choose an $m \in \mathbb{N}$ with the property that for any interval $I$ the set $q(I)$ is a union of at most $m$ MSI. Pick $l \in \mathbb{N}$ and the interval $I$ satisfying the following conditions:

$$
q(I) \subset \bigcup_{n>2 l m} \bigcup_{k=1}^{n} J_{k}^{(n)},
$$

and

$$
\text { card } I=2 \mathrm{~lm} \text {. }
$$




\section{CONVERGENT AND DIVERGENT PERMUTATIONS}

Then, according to the assumption, the set $q(I)$ contains an interval $U$ which cardinality is $\geqslant m^{-1}$ card $I=2 l$. By condition (2.1), there exists a subinterval $J$ of the interval $U$ such that

$$
J \subset J_{k}^{(n)} \quad \text { for some } k \in\{1, \ldots, n\} \text { and for some } n>2 l m,
$$

and

$$
\text { card } J \geqslant l \text {. }
$$

However, by (2.3) and by the definition of $p$, the inequality

$$
p(a)-p(b) \geqslant n
$$

holds whenever $a, b \in J$ and $a>b$ (more precisely, we have $p(a)-p(b)=(a-b) n$ ). Hence, by (2.3) and (2.2), we obtain

$$
p(a)-p(b)>\operatorname{card} I
$$

for any two $a, b \in J, a>b$. This implies that the set $p q(I)$ is a union of at least card $(J)$ MSI because the set $p q(I)$ has the cardinality precisely equal to card $I$ and the following inclusion $p(J) \subset p q(I)$ holds. In other words, since for any two different $a, b \in J$, if $a>b$, then

$$
(p(b), p(a)) \backslash p q(I) \neq \emptyset
$$

and $\operatorname{card} p q(I)=\operatorname{card} I$, the set $p q(I)$ must be a union of at least $\operatorname{card}(J)$ MSI. Since we have not made any assumptions on $l$, we learn by (2.4) that $p q \in \mathfrak{D}$. But, $p \in \mathfrak{D} \mathfrak{D}$, which is clear from the definition of $p$. Thus, we get that $p q \in \mathfrak{D} \mathfrak{D}$ (cf. [3, Theorems 2.4 and 2.6]).

Now, we will show that $\mathfrak{C} \circ p \subset \mathfrak{D} \mathfrak{D}$. Suppose $q p \in \mathfrak{C}$ for some $q \in \mathfrak{C}$. Let $m \in \mathbb{N}$ be chosen in such a way that for each interval $I$ any of the following sets $q(I)$ and $q p(I)$ is a union of at most $m$ MSI. Additionally, let a number $n \in \mathbb{N}, n>2 m(m+1)$ be given. Then any of the sets $q\left(J_{k}^{(n)}\right)$ for $k=1, \ldots, n$, contains an interval $\Omega_{k}$ having the cardinality $\geqslant n / m$. Obviously, the intervals $\Omega_{k}, k=1, \ldots, n$, are pairwise disjoint. It follows from the definition of $p$ that

$$
\operatorname{card}\left(J_{k}^{(n)} \cap p\left(J_{i}^{(n)}\right)\right)=1
$$

for any two indices $i, k \in\{1,2, \ldots, n\}$. We also have the following inequality

$$
\operatorname{card}\left(\Omega_{k} \cap q p\left(J_{i}^{(n)}\right)\right) \leqslant 1
$$

for any $i, k \in\{1,2, \ldots, n\}$. On the other hand, since

$$
p(J)=J \quad \text { and } \quad \bigcup_{k=1}^{n} \Omega_{k} \subset q(J) \quad \text { for } \quad J=\bigcup_{k=1}^{n} J_{k}^{(n)},
$$


there is an index $i \in\{1, \ldots, n\}$ such that

$$
\operatorname{card}\left(q p\left(J_{i}^{(n)}\right) \cap \bigcup_{k=1}^{n} \Omega_{k}\right) \geqslant n^{-1} \operatorname{card}\left(\bigcup_{k=1}^{n} \Omega_{k}\right) \geqslant m^{-1} n>2 m+2 .
$$

Indeed, otherwise we would have (this remark concerns only the first inequality from the above ones)

$$
\operatorname{card}\left(q p\left(J_{i}^{(n)}\right) \cap \bigcup_{k=1}^{n} \Omega_{k}\right)<n^{-1} \operatorname{card}\left(\bigcup_{k=1}^{n} \Omega_{k}\right),
$$

for each $i=1,2, \ldots, n$, that is

$$
\begin{aligned}
& \sum_{i=1}^{n} \operatorname{card}\left(q p\left(J_{i}^{(n)}\right) \cap \bigcup_{k=1}^{n} \Omega_{k}\right) \\
& =\operatorname{card}\left(\bigcup_{i=1}^{n} q p\left(J_{i}^{(n)}\right) \cap \bigcup_{k=1}^{n} \Omega_{k}\right)=\operatorname{card}\left(q p(J) \cap \bigcup_{k=1}^{n} \Omega_{k}\right) \\
& =\operatorname{card}\left(q(J) \cap \bigcup_{k=1}^{n} \Omega_{k}\right)=\operatorname{card}\left(\bigcup_{k=1}^{n} \Omega_{k}\right) \\
& <\sum_{i=1}^{n} n^{-1} \operatorname{card}\left(\bigcup_{k=1}^{n} \Omega_{k}\right)=\operatorname{card}\left(\bigcup_{k=1}^{n} \Omega_{k}\right)
\end{aligned}
$$

which is impossible.

Hence, by (2.5) and by the estimation card $\Omega_{k} \geqslant 2$ for every index $k=1, \ldots, n$, it is not difficult to conclude that the set $q p\left(J_{i}^{(n)}\right)$ is a union of at least $(m+1)$ MSI. This contradicts our assumption and therefore, $\mathfrak{C} \circ p \subset \mathfrak{D}$. Since $p \in \mathfrak{D} \mathfrak{D}$, then the relation $\mathfrak{C} \circ p \subset \mathfrak{D D}$ is obvious.

Remark 2.4. We note that from the construction of permutation $p$ in Example 1 it follows that the family $\mathfrak{P} \backslash(\mathfrak{C D} \circ \mathfrak{D C} \cup \mathfrak{C D} \circ \mathfrak{C D})$ has the power of continuum. More precisely, by the slightly modified construction of permutation $p$, we will see that it enables us to obtain the family of such permutations having the power of continuum.

For this purpose, let us assign the permutation $p=p(r)$ to each increasing sequence $r=\left\{r_{n}\right\}_{n=1}^{\infty}$ of positive integers, definition of which will be changed in comparison to the original definition of permutation $p$ from Example 1, in the following way. We take that $\operatorname{card} J_{k}^{(n)}=r_{n}$, for any $k, n \in \mathbb{N}, k \leq n$, and, additionally, we set

$p\left(i-1+\min J_{k}^{(n)}\right)=k-1+\min J_{i}^{(n)} \quad$ for any $i, k=1,2, \ldots, r_{n} \quad$ and $\quad n \in \mathbb{N}$. 


\section{CONVERGENT AND DIVERGENT PERMUTATIONS}

Since the family of all such sequences $\left\{r_{n}\right\}_{n=1}^{\infty}$ has the power of continuum, the proof is completed.

Now, we present the fourth theorem from among the announced ones. It may be commented in the following way: the set $\mathfrak{C} \mathfrak{C}$ is very big, indeed, also structurally (we note that card $(\mathfrak{C} \mathfrak{C})=\mathfrak{c}$ ), because we have (see [3, Theorem 2.4]). From this theorem it results that permutations from the family $\mathfrak{C} \mathfrak{C}$ can completely change the combinatoric nature of composed permutations.

TheOREM 2.5. For any $p \in(\mathfrak{C D} \cup \mathfrak{D D})$ and $q \in \mathfrak{D}$ there exist permutations $\varrho, \sigma \in \mathfrak{C} \mathfrak{C}$ such that $p \varrho q \in \mathfrak{D} \mathfrak{D}$ and $q \sigma p \in \mathfrak{D} \mathfrak{D}$. In other words, the following relations hold

hold.

$$
(p \mathfrak{C} \mathfrak{C} q) \cap \mathfrak{D} \mathfrak{D} \neq \varnothing \quad \text { and } \quad(q \mathfrak{C} \mathfrak{C} p) \cap \mathfrak{D} \mathfrak{D} \neq \varnothing
$$

P r o of. First, we will construct the permutation $\varrho$. Suppose that the sequences $I_{n}$ and $J_{n}, n \in \mathbb{N}$, of intervals of positive integers have been selected in such a way that the following conditions are satisfied:

$$
1+\left(I_{n} \cup q\left(I_{n}\right)\right)<J_{n} \cup p^{-1}\left(J_{n}\right)<\left(I_{n+1} \cup q\left(I_{n+1}\right)\right)-1
$$

any of the following sets $q\left(I_{n}\right)$ and $p^{-1}\left(J_{n}\right)$ is a union

$$
\text { of at least } 2 n \text { MSI for any } n \in \mathbb{N} \text {. }
$$

Put

$$
q\left(I_{n}\right)=\bigcup_{i=1}^{k(n)} G_{n}^{(i)} \text { and } p^{-1}\left(J_{n}\right)=\bigcup_{i=1}^{l(n)} H_{n}^{(i)}
$$

where $G_{n}^{(i)}, i=1, \ldots, k(n)$, as well as $H_{n}^{(i)}, i=1, \ldots, l(n)$, are sequences of mutually separated intervals. We will denote by $a_{n}^{(i)}, i=1, \ldots, k(n)$, and by $b_{n}^{(i)}$, $i=1, \ldots, l(n)$, the increasing sequences of all elements of the sets $\left\{p\left(\max G_{n}^{(i)}\right)\right.$ : $i=1, \ldots, k(n)\}$ and $\left\{q^{-1}\left(\max H_{n}^{(i)}\right): i=1, \ldots, l(n)\right\}$, respectively. Now, for all even indices $i \in\{1,2, \ldots, k(n)\}$ and $j \in\{1,2, \ldots, l(n)\}, n \in \mathbb{N}$, we define the permutation $\varrho$ as a product of the transposition of the elements

and

$$
p^{-1}\left(a_{n}^{(i)}\right) \text { and } 1+p^{-1}\left(a_{n}^{(i)}\right)
$$

$$
q\left(b_{n}^{(j)}\right) \text { and } 1+q\left(b_{n}^{(j)}\right),
$$

respectively. By condition (2.6), this definition is correct. It is not difficult to verify that $\varrho \in \mathfrak{C} \mathfrak{C}$ and that $\varrho=\varrho^{-1}$. Moreover, the definitions of the sequences $a_{n}^{(i)}$, $i=1, \ldots, k(n)$, and of the permutation $\varrho$ imply that

$$
a_{n}^{(i)} \in p \varrho q\left(I_{n}\right) \quad \text { if and only if the index } i \text { is odd }
$$

for each $i=1, \ldots, k(n)$ and for any $n \in \mathbb{N}$. 
Thus the set $p \varrho q\left(I_{n}\right)$ is a union of at least $2^{-1} k(n) \geqslant($ by (2.7) $) \geqslant n$ MSI, because the sequence $a_{n}^{(i)}, i=1, \ldots, k(n)$, is increasing. Analogously, as above, it may be shown that the set $q^{-1} \varrho^{-1} p^{-1}\left(J_{n}\right)$ is a union of at least $2^{-1} l(n) \geqslant$ (by (2.7) $) \geqslant n$ MSI. Thus, $p \varrho q \in \mathfrak{D} \mathfrak{D}$, as desired.

Now, we proceed to define the permutation $\sigma$. We first pick two increasing sequences $I_{n}$ and $J_{n}, n \in \mathbb{N}$, of intervals of $\mathbb{N}$ such that

$$
I_{n}<J_{n}<I_{n+1}, \quad n \in \mathbb{N},
$$

and for every $n \in \mathbb{N}$ there exist two increasing sequences of intervals

$$
G_{n}^{(i)} \subset I_{n} \quad \text { and } \quad H_{n}^{(i)} \subset J_{n} \quad \text { for } \quad i=1, \ldots, 5,
$$

such that

(a) any of the sets $q\left(H_{n}^{(1)}\right)$ and $p^{-1}\left(G_{n}^{(1)}\right)$ is a union of at least $n$ MSI,

(b) $G_{n}^{(4)}>q^{-1}\left(G_{n}^{(2)}\right)>G_{n}^{(1)}$ and $H_{n}^{(4)}>p\left(H_{n}^{(2)}\right)>H_{n}^{(1)}$,

(c) the sets $q^{-1}\left(G_{n}^{(2)}\right)$ and $p\left(H_{n}^{(2)}\right)$ contain the intervals $G_{n}^{(3)}$ and $H_{n}^{(3)}$, respectively, such that

$$
\left|G_{n}^{(3)}\right|=\left|G_{n}^{(1)}\right| \quad \text { and } \quad\left|H_{n}^{(3)}\right|=\left|H_{n}^{(1)}\right|,
$$

(d) $\left|G_{n}^{(4)}\right|=\min G_{n}^{(3)}-\min q^{-1}\left(G_{n}^{(2)}\right)$ and $\left|H_{n}^{(4)}\right|=\min H_{n}^{(3)}-\min p\left(H_{n}^{(2)}\right)$,

(e) $\left|G_{n}^{(5)}\right|=\max q^{-1}\left(G_{n}^{(2)}\right)-\max G_{n}^{(3)}$ and $\left|H_{n}^{(5)}\right|=\max p\left(H_{n}^{(2)}\right)-\max H_{n}^{(3)}$,

(f) $q\left(H_{n}^{(i)}\right)>q\left(H_{n}^{(1)}\right)$ whenever $H_{n}^{(i)} \neq \varnothing$ and $p^{-1}\left(G_{n}^{(i)}\right)>p^{-1}\left(G_{n}^{(1)}\right)$ whenever $G_{n}^{(i)} \neq \varnothing$ for $i=4,5$ in both cases.

Observe that, by (d) and (e), the intervals $G_{n}^{(i)}, H_{n}^{(i)}, i=4,5$, may be empty. Now, using the above arguments, we define $\sigma$ as an increasing mapping of the subsequent intervals

$$
\begin{gathered}
A_{n}^{(3)}, A_{n}^{(4)}, A_{n}^{(5)}, A_{n}^{(1)} \\
{\left[\max A_{n}^{(3)}+1, \max \gamma\left(A_{n}^{(2)}\right)\right], \quad\left[\min \gamma\left(A_{n}^{(2)}\right), \min A_{n}^{(3)}-1\right]}
\end{gathered}
$$

onto the intervals

$$
\begin{gathered}
A_{n}^{(1)}, \quad\left[\min \gamma\left(A_{n}^{(2)}\right), \min A_{n}^{(3)}-1\right], \quad\left[\max A_{n}^{(3)}+1, \max \gamma\left(A_{n}^{(2)}\right)\right], \\
A_{n}^{(3)}, A_{n}^{(4)}, A_{n}^{(5)}
\end{gathered}
$$

in the specified order (i.e., $A_{n}^{(3)} \rightarrow A_{n}^{(1)}$, etc., the condition (c) is needed here) for every $n \in \mathbb{N}$, where $A=G$ or $H$ and $\gamma=q^{-1}$ or $p$, respectively. 


\section{CONVERGENT AND DIVERGENT PERMUTATIONS}

Moreover, we put $\sigma(n)=n$ for all other $n \in \mathbb{N}$. Then, the following equalities holds

$$
\begin{array}{r}
q \sigma p\left(H_{n}^{(2)}\right)=q\left(H_{n}^{(1)} \cup H_{n}^{(4)} \cup H_{n}^{(5)}\right), \\
p^{-1} \sigma^{-1} q^{-1}\left(G_{n}^{(2)}\right)=p^{-1}\left(G_{n}^{(1)} \cup G_{n}^{(4)} \cup G_{n}^{(5)}\right)
\end{array}
$$

holds. Therefore, according to assumptions (a) and (f), any of the two following sets $q \sigma p\left(H_{n}^{(2)}\right)$ and $p^{-1} \sigma^{-1} q^{-1}\left(G_{n}^{(2)}\right)$ is a union of at least $n$ MSI. Hence, $q \sigma p \in$ $\mathfrak{D D}$, as claimed.

Remark 2.6. From the proof presented above we see that if $p \in(\mathfrak{C} \mathfrak{D} \cup \mathfrak{D D})$ and $q \in \mathfrak{D}$, then there exists a permutation $\varrho \in \mathfrak{C} \mathfrak{C}$ such that

$$
p \varrho q \in \mathfrak{D D} \text { and } \varrho^{2}=\operatorname{id}(\mathbb{N}) \text {. }
$$

The last condition means that $\varrho$ is a product of disjoint transpositions.

Theorem 2.7. Let $p \in \mathfrak{P}$. Then $p \mathfrak{D} p^{-1} \subset \mathfrak{D}$ if and only if $p \in \mathfrak{C} \mathfrak{C}$ and $p \mathfrak{D D} p^{-1} \subset \mathfrak{D D}$ if and only if $p \in \mathfrak{C} \mathfrak{C}$. When $p \in \mathfrak{C} \mathfrak{C}$, then $p \mathfrak{D} p^{-1}=\mathfrak{D}$ and $p \mathfrak{D} \mathfrak{D} p^{-1}=\mathfrak{D} \mathfrak{D}$.

Pr o of. In view of the previous theorem, if $p \in \mathfrak{P} \backslash \mathfrak{C} \mathfrak{C}$, then there is a permutation $\sigma \in \mathfrak{C} \mathfrak{C}$ such that $p^{-1} \sigma p \in \mathfrak{D} \mathfrak{D}$. Hence, $\sigma \in\left(p \mathfrak{D} \mathfrak{D} p^{-1}\right)$, i.e., $\mathfrak{C} \cap\left(p \mathfrak{D} \mathfrak{D} p^{-1}\right) \neq \varnothing$. On the other hand, if $p \in \mathfrak{C} \mathfrak{C}$ then, using of [3. Theorem 2.6], we obtain $p \mathfrak{D} p^{-1}=\mathfrak{D}$ and $p \mathfrak{D} \mathfrak{D} p^{-1}=\mathfrak{D} \mathfrak{D}$.

\section{Acknowledgements}

The author would like to thank J. W ł od a r z for his valuable discussions and suggestions as well as the referee for important remarks and advice which enable the author to improve presentation of the paper and clarity of the proofs.

\section{REFERENCES}

[1] KRONROD, A.S.: On permutation of terms of numerical series, Mat. Sb. 1860 (1946), 237-280. (In Russian)

[2] WITUŁA, R.: On the set of limit points of the partial sums of series rearranged by a given divergent permutation, J. Math. Anal. Appl. 362 (2010), 542-552.

[3] WITUŁA, R.: Algebraic properties of the convergent and divergent permutations, Filomat (in review).

[4] WITUŁA, R.: Permutations preserving the sum of real convergent series, Cent. Eur. J. Math. 11 (2013), 956-965.

[5] WITUŁA, R.: The family $\mathfrak{F}$ of permutations of $\mathbb{N}$, Math. Slovaca (submitted).

[6] WITUŁA, R.-SŁOTA, D.-SEWERYN, R.: On Erdös' theorem for monotonic subsequences, Demonstratio Math. 40 (2007), 239-259. 


\section{ROMAN WITUŁA}

[7] WITUŁA, R.: The Riemann derangement theorem and divergent permutations, Tatra Mt. Math. Publ. 52 (2012), 75-82.

Received October 19, 2012

Institute of Mathematics

Silesian University of Technology

Kaszubska 23

PL-44-100 Gliwice

POLAND

E-mail: roman.witula@polsl.pl 